\title{
Magnetron Frequency Twinning
}

\author{
Richard G. Carter, Member, IEEE, Elaine M. Ball, and Michael B. Brady
}

\begin{abstract}
Pulsed magnetron oscillators sometimes show an instability known as "twinning" in which the spectrum shows two, or more, closely spaced peaks. This phenomenon is undesirable and can lead to problems when it occurs in magnetrons used in radars. An experimental investigation of twinning is described from which conclusions are drawn about the design parameters that affect it. It has been shown that changes in factors, such as the magnetic field strength, the external match, the cathode coating, and the cathode temperature, can be used to reduce the probability of twinning or to move the point at which it occurs outside the normal operating range of the tube. Possible theoretical explanations are discussed.
\end{abstract}

Index Terms-Frequency twinning, magnetrons, microwave tubes.

\section{INTRODUCTION}

A N INSTABILITY, known as twinning [1], is exhibited by some magnetrons under certain operating conditions. This instability causes the magnetron to oscillate at two (or sometimes more) frequencies. Figs. 1 and 2 show examples of the frequency spectrum of a low-power X-band magnetron. Both spectra were produced by the same tube but at different anode voltages. Fig. 1 shows the desired spectrum when the tube is not twinning. When the tube is twinning (Fig. 2), the spectrum shows two peaks separated by only a few megaHertz. Although this phenomenon has been known for many years, it has received surprisingly little attention in the literature and we have not been able to find any more recent papers on it. Not all magnetrons suffer from twinning, and in many cases, it does not occur within the normal operating range of the tube. When it does occur, however, it can cause a significant number of tubes to be rejected during manufacture. It may also develop during the life of a tube as a result of aging processes with the consequence that the system in which it is installed does not perform as expected. This paper describes the results of an experimental investigation that aimed to gain a greater understanding of twinning and of the factors affecting it.

\section{Magnetron Performance}

A magnetron is a diode that conducts very little current as the anode voltage is increased from zero until the threshold voltage is reached at which oscillations begin. The oscillations

Manuscript received October 6, 1999; revised March 8, 2000. This work was supported by EEV Ltd. (now Marconi Applied Technologies) and the Engineering and Physical Sciences Research Council, under a Cooperative Research Grant.

R. G. Carter is with the Engineering Department, Lancaster University, LA1 4YR Lancaster, U.K.

E. M. Ball was with the Engineering Department, Lancaster University, LA1 4YR Lancaster, U.K. She is now with Hewlett-Packard, Bristol B534 8QZ, U.K. M. B. Brady is with Marconi Applied Technologies, Chelmsford CM1 2QU, U.K.

Publisher Item Identifier S 0093-3813(00)06056-2.

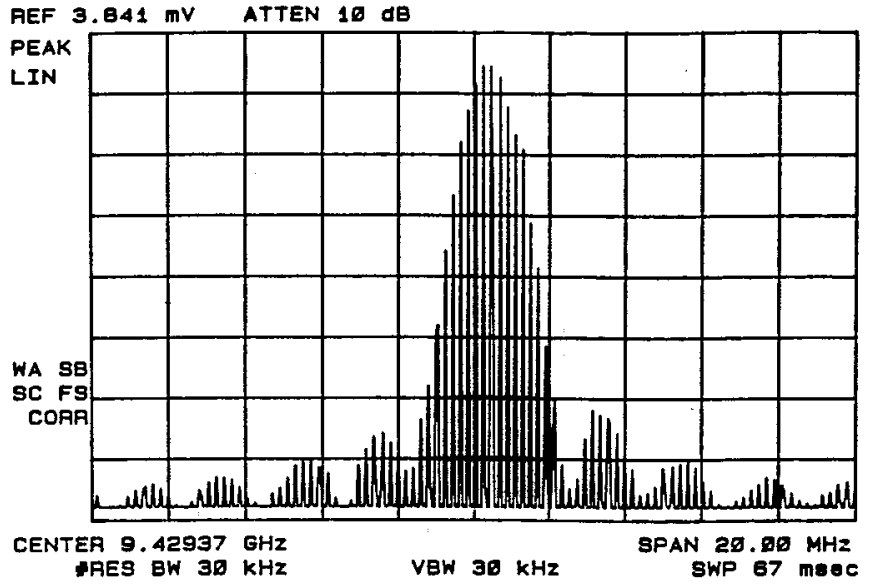

Fig. 1. Normal magnetron spectrum.

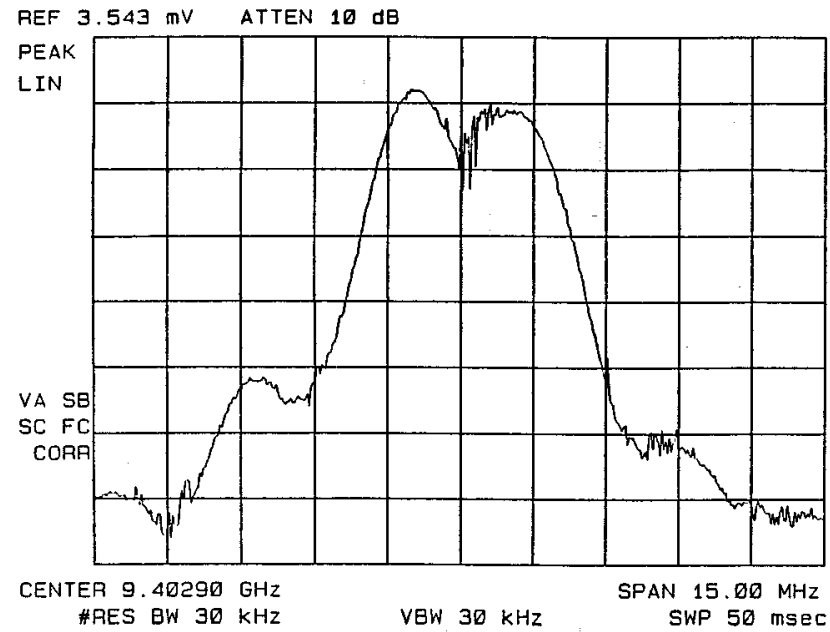

Fig. 2. Magnetron spectrum showing twinning.

then build rapidly until the output power saturates. Once the tube is oscillating the anode voltage is not found to vary very much over a wide range of anode currents. Fig. 3 shows the relationship between the anode voltage and current for a typical tube. It can be seen that the current/voltage relationship also depends on the strength of the axial magnetic field in the interaction region. The lines in the current/voltage plot commonly exhibit discontinuities that are known as "Gauss line discontinuities" [1].

The frequency of oscillation of a magnetron is determined by the resonant frequency of the anode in its $\pi$-mode modified by the effects of electron loading. These cause the frequency to change as the anode current changes, a phenomenon known as frequency pushing. Fig. 4 shows a typical frequency pushing curve for a modern magnetron. The tube is designed to be operated in the region to the right of the peak of the curve so that the 


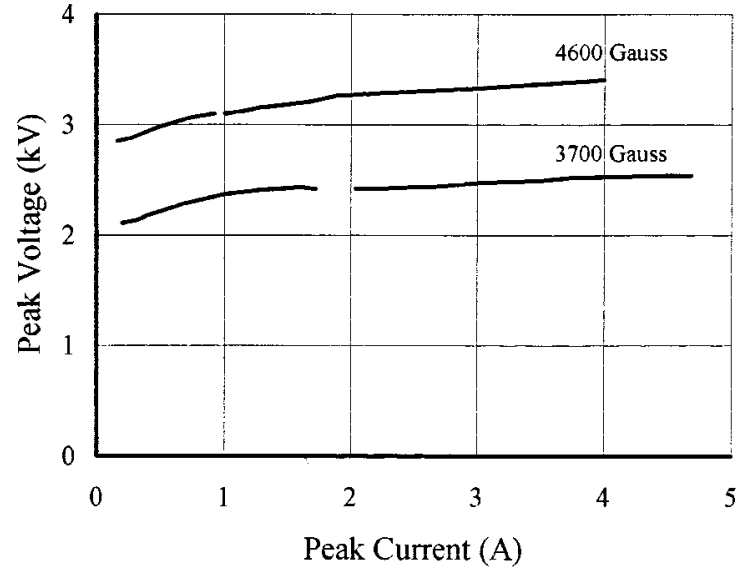

Fig. 3. Typical current/voltage curves showing discontinuities associated with twinning.

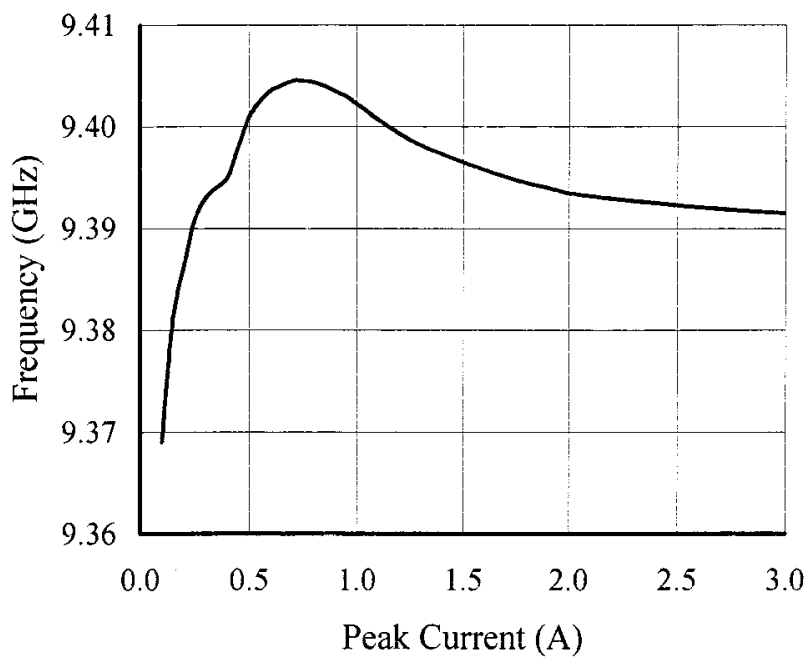

Fig. 4. Typical frequency pushing curve.

frequency is not strongly dependent on the current. The kink in the curve just to the left of the peak is seen in some tubes, and twinning commonly occurs close to this point. Twinning may also occur at higher currents within the normal operating range of the tube. It is observed that even in tubes that do not twin, there is a marked change in the spectrum in the vicinity of the kink in the pushing curve. This is generally attributed to the rapid change in the slope of the pushing curve in this region. The frequency of oscillation is also affected by the amplitude and phase of any external mismatch. This changes the reactive loading on the anode resulting in frequency pulling.

\section{OBSERVATIONS OF TWINNING}

An extensive series of experimental observations was carried out on standard and modified low-power X-band pulsed magnetrons. The permanent magnets were removed from the tubes to allow them to be operated in an electromagnet so that the magnetic field strength could be varied. It was also necessary to modify the modulator so that fine adjustments could be made to the anode voltage because twinning commonly occurs over a very narrow range of operating conditions. Observations were made of the current, voltage, and radio frequency (RF) pulse

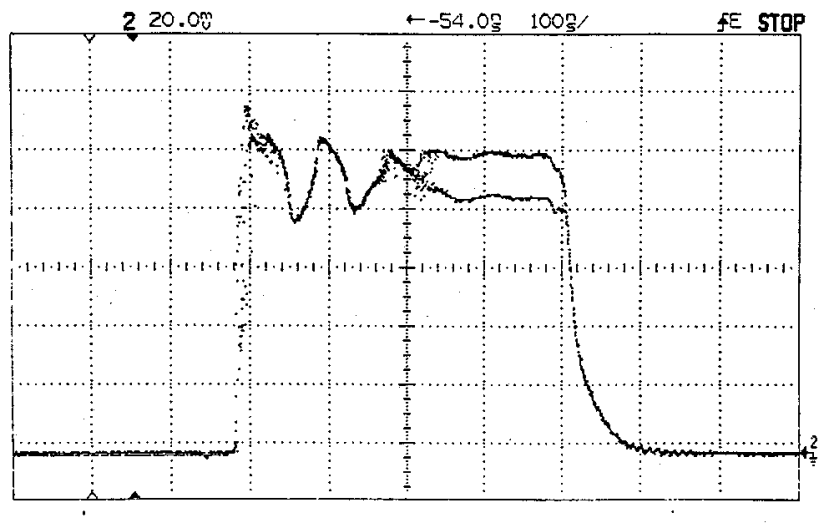

Fig. 5. Typical RF pulse when the tube is twinning.

waveforms and of the frequency spectrum. The onset of twinning was observed in the spectrum and in the splitting of the RF output pulse, as shown in Fig. 5. When a sequence of RF output pulses was recorded, it was found that there were two pulse shapes that occurred randomly with, occasionally, some intermediate shape, as shown in Fig. 6. From this, it appears that the magnetron may switch during a pulse between two states that have different anode currents and frequencies of oscillation. It is important to distinguish between these states and the modes of oscillation associated with different resonances of the anode. When a change of the anode mode takes place, the magnetron is said to be "moding" or having "missing pulses," and the effects are much larger than are those associated with twinning.

Additional studies were carried out using a much wider range of magnetron types to find out which design parameters might be relevant to controlling the occurrence of twinning. This study showed no clear correlations, except that twinning was much more likely to be observed in tubes for which the ratio of the electron cyclotron frequency to the frequency of oscillation lay in the range 0.75 to 1.25 .

\section{A. Dependence of Twinning on the Voltage Pulse}

When a tube twins, it does so over one or more narrow ranges of the anode voltage, and some care is needed to make sure that an occurrence of twinning is not missed. Twinning may coincide with a Gauss Line discontinuity, but this is not necessarily the case. Not all discontinuities are associated with twinning, and twinning may occur where there is no observable discontinuity. In the latter case, it may be that there is a discontinuity that is too small to observe. The frequency difference between the twin states was found to be related to the change in the anode current, and to the slope of the frequency pushing curve at that point, so that large current changes tended to be associated with relatively larger frequency jumps. As the anode voltage was increased through a region of twinning, it was observed that there was a steady change in the proportions of the pulses at the two frequencies.

For the high current twin, it was found that the split in the current pulse occurred somewhere close to the center of the pulse. When the pulse was then shortened, the twinning disappeared. This is consistent with the view that the tube is switching from one state to another during the pulse. For the low current twin, 

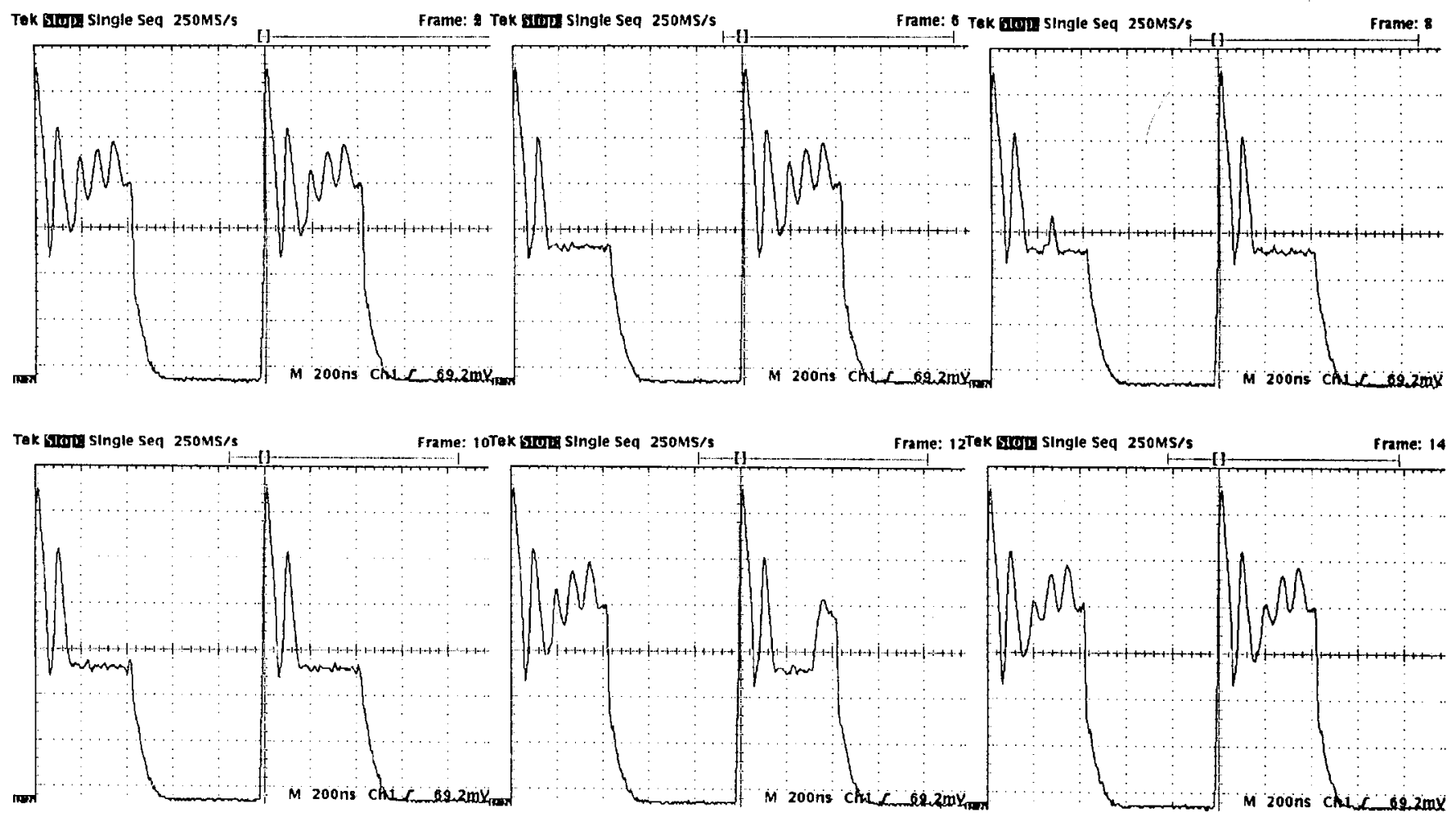

Fig. 6. Example of a sequence of RF output pulses when the tube is twinning.

on the other hand, the split in the current pulse appeared to occur at, or near, the beginning of the pulse. The change of state must, therefore, be happening very rapidly early in the pulse, for this type of twinning, or else the frequency must be changing from pulse to pulse. If twinning is associated with changes between different states of the electron cloud within the tube, it might be expected that it would be influenced by changes in the impedance of the modulator. We observed changes in the frequency jump associated with the twin as a result of changes in the modulator impedance, but we were not able to eliminate twinning by this means.

\section{B. Dependence of Twinning on the Magnetic Field}

An investigation was carried out into the effect on twinning of changes in the magnetic field in the interaction region. The range of field strengths that could be used was limited to those for which the magnetron would operate stably. Fig. 7 shows the frequency pushing curves at three different magnetic fields for a tube that twinned at both low and high currents. The lower current at which twinning occurs did not change much with magnetic field, whereas the higher current varied widely with changes in the field. This suggests that there may be at least two different types of twinning with different underlying physical mechanisms. In this tube, it would be possible to keep the normal operating range free from twinning by a suitable choice of the magnetic field. The ratios of the cyclotron frequency to the frequency of oscillation for the three curves are 1.11, 1.26, and 1.38. Thus, the highest curve corresponds to a value of this parameter for which we have already observed that twinning is less likely to occur in the normal operating range of the tube.

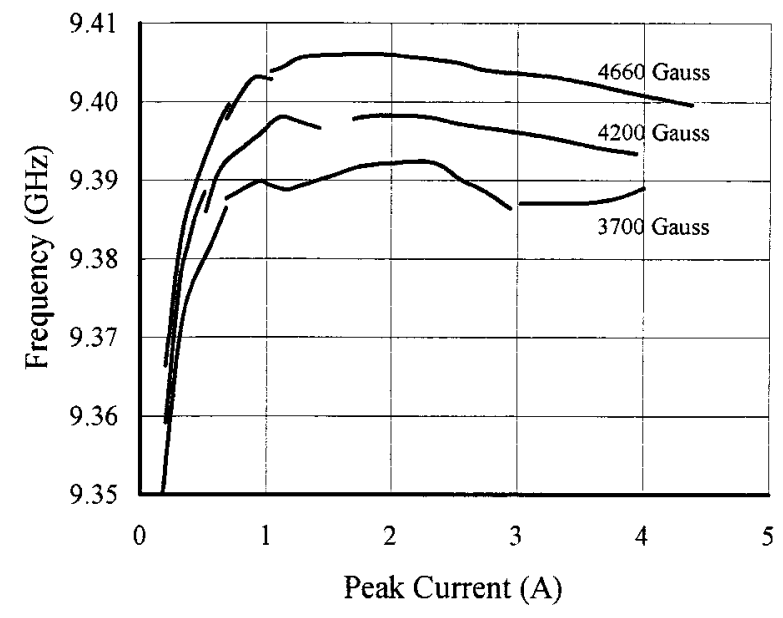

Fig. 7. Frequency pushing curves showing the dependence of twinning on magnetic field.

\section{Dependence of Twinning on the Cathode}

It was known from earlier studies that twinning could be affected by changes in the composition of the cathode coating. Experiments were carried out in which the heater power was changed. This was also found to have a marked effect on the twinning, but in ways that differed appreciably between nominally identical tubes. In some cases, a change in the heater power caused additional twinning frequencies to appear. There was also a change in the frequency jump, but the change was an increase in some tubes and a decrease in others. The change in the heater power generally moved the twin to a different oper- 


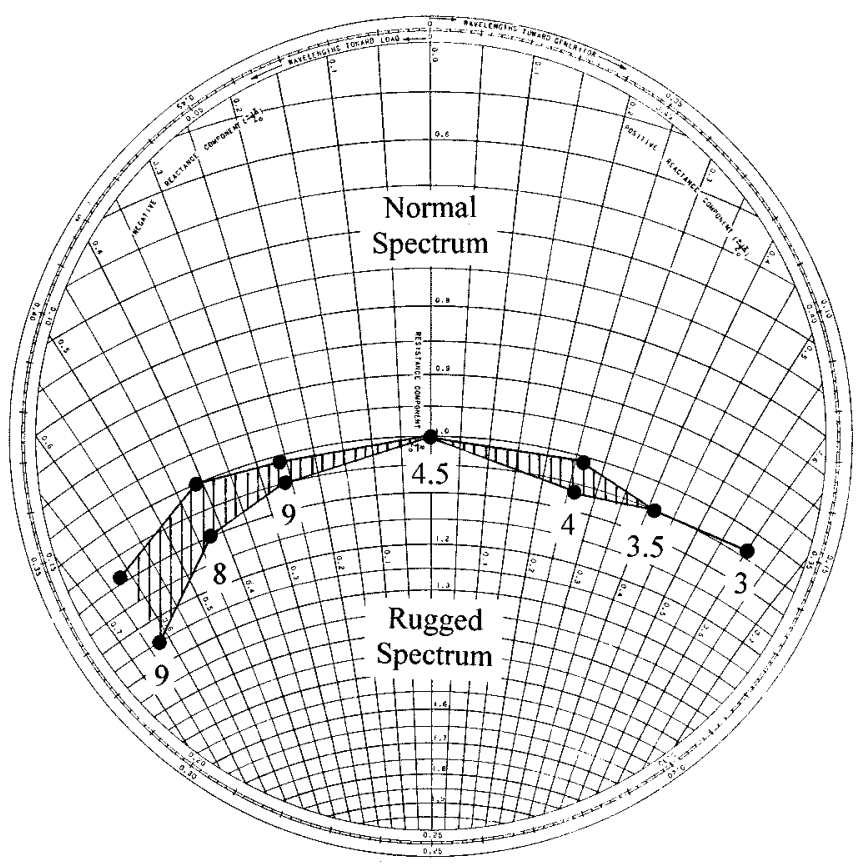

Fig. 8. Rieke diagram showing the effect of external mismatch on twinning. The shaded region shows where the tube was twinning for different values of the VSWR. The frequency difference between the pairs of twinning states is shown in megaHertz.

ating point, and it affected the frequency through changes in the temperature of the anode.

The concentricity of the cathode is also known to have an effect on twinning. On occasion, it is possible to improve the performance of a tube by moving the cathode sideways. Unfortunately, it is not easy to tell in any individual case whether the performance has been improved by increasing or decreasing the concentricity. Experimental tubes were manufactured in which the cathode was deformed so that its cross section was no longer circular, but this was found to make little difference to the twinning.

\section{Dependence of Twinning on the External Match}

A variable mismatch whose amplitude and phase could be varied independently was inserted in the output waveguide. The tube was first adjusted to be twinning without any mismatch, the mismatch was introduced, and the behavior of the tube noted as the amplitude and phase were varied. Fig. 8 shows a typical set of results in which the reference plane was chosen so that the points at which twinning was observed lay on the circle corresponding to a mismatch having a real part equal to unity. For each VSWR, the tube twinned in the shaded region of the diagram with the frequency differences between the twinning states in megaHertz as marked. The twinning could, therefore, be stopped by changing the mismatch, though this presumably moved it to a different operating point rather than eliminating it completely. This was confirmed by setting the anode current to values above and below the twinning point when it was found that the tube could be induced to twin by adjusting the external match. In each case, the twinning region divided the chart into two regions in which the shapes of the spectra were different and corresponded to those observed at anode currents above and below the twinning point.

\section{DISCUSSION}

It seems probable that twinning is caused by changes in the loading of the anode by electrons. The existence of twinning, and of Gauss Line discontinuities, at more than one operating point suggests that there are a number of possible states, each of which:

1) is stable over a certain range of operating conditions;

2) has its own Gauss Line;

3) has its own spectrum.

Both twinning and Gauss Line discontinuities would, therefore, occur under those conditions in which there was a change from one stable state to another. Because the oscillation of a magnetron starts up from random noise, it would follow that when two states were equally stable, oscillation might occur in either of them with random occurrence from pulse to pulse. In other cases, the starting conditions might be such as to select an unstable state with the possibility of a jump to a more stable state during the pulse. It is not clear, however, whether the twinning observed at different currents is associated with one, or more than one, underlying physical phenomenon.

Schumacher [1] suggested that twinning was caused by axial variations in the electron cloud associated with axial variations in the strength of the magnetic field. His hypothesis was that there could be two, or more, elementary magnetrons, each of which would have a slightly different frequency because of the different electron loading of the anode. The frequency would then be determined by whichever of these elementary magnetrons represented the preferred state. We used computer simulation to investigate the uniformity of the magnetic field in the magnetrons used in our experiments. It was concluded that the departures of the field from uniformity were unlikely to be big enough for Schumacher's hypothesis to explain the mechanism of twinning in these tubes.

Another possible hypothesis is that twinning is caused by multipactor discharges within the magnetron. The observations made by Vaughan on a low-voltage continuous wave magnetron [2] showed that multipactor discharges are not uncommon in such tubes. Discharges were observed between the vane tips and between the vane tips and the pole pieces. Multipactor discharges start and stop abruptly as the operating conditions are changed, and crossed-field discharges occur over wide ranges of operating conditions [3]. It is, therefore, possible that the high current twin, which is strongly affected by the magnetic field, as shown in Fig. 7, is associated with the onset of such a discharge. However, it is not clear how the conditions for multipactor discharge on the anode would be affected by changes in the cathode coating, temperature, or concentricity. Thus, multipactor discharges may be responsible for the high-current twin, but cannot explain the low-current twin.

Recent work by McDowell [4] has shown striking differences between the behavior of the electrons within the magnetron in simulations with different assumptions about the electron emission from the cathode. All observations of twinning reported 
in this paper were made on tubes whose normalized parameters were similar to those of the 4J50/4J52 simulated by McDowell. In our experiments, the twinning occurred at cathode current densities exceeding $20 \mathrm{~A} . \mathrm{cm}^{-2}$, and the tubes were, therefore, in McDowell's secondary emission dominated state. McDowell showed that in this state, the electron cloud had a region of low-charge density that moved through the spokes. It was found that the characteristic phenomena of the secondary emission dominated state were not strongly affected by changes in the secondary electron emission coefficient of the cathode. At first sight, this would seem to rule out these fluctuations of the inner space charge as a cause of twinning. However, we would observe that twinning is associated with only small changes in the performance of a magnetron and that all our observations are consistent with the suggestion that it is dependent in some way on secondary electron emission from the cathode. Because twinning occurs over only a very small range of operating conditions, it is unlikely that McDowell's simulation would have revealed just those conditions under which twinning occurs. It would, therefore, be interesting to see whether the mechanism of twinning could be demonstrated using computer simulation.

\section{CONCLUSION}

The experimental study carried out has added considerably to our knowledge of the phenomenon of twinning. Design parameters having a critical effect on the occurrence of twinning include the choice of magnetic field, of cathode coating, and of cathode temperature. By careful choice of these, it is possible, in many cases, to eliminate twinning or to move the points at which it occurs outside the normal operating range of the tube. Possible explanations of twinning have been advanced that are broadly consistent with our experimental observations. Further research is needed to investigate these hypotheses in detail.

\section{ACKNOWLEDGMENT}

The authors would like to thank N. Nicholls for helpful discussions and Marconi Applied Technologies for permission to publish this paper.

\section{REFERENCES}

[1] C. R. Schumacher, "Spectrum shape," in Crossed-Field Microwave Devices, E. Okress, Ed. New York, NY: Academic Press, 1961, vol. 2.
[2] J. R. M. Vaughan, "Observations of multipactor in magnetrons," IEEE Trans. Electron Devices, vol. ED-15, no. 11, pp. 883-889, November 1968.

[3] — , "Multipactor," IEEE Trans. Electron Devices, vol. ED-35, no. 6, pp. 1172-1180, July 1988.

[4] H. L. McDowell, "Magnetron simulations using a moving wavelength computer code," IEEE Trans. Plasma Sci., vol. PS-26, pp. 733-754, June 1998.

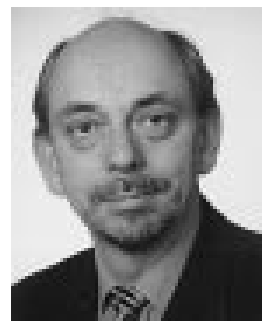

Richard G. Carter (M'97) graduated in physics from the University of Cambridge in 1965 and received the Ph.D. from the University of Wales in 1968 for work on the propagation of waves on neutralized ion beams. From 1968 to 1972, he worked on high power traveling-wave tubes as a Development Engineer at the English Electric Valve Company, Ltd. He joined the Engineering Department of the University of Lancaster as a Lecturer in 1972 and was promoted to Senior Lecturer in 1986 and Professor of electronic engineering in 1996.

He is currently Dean of the Faculty of Applied Sciences. His research interests include electromagnetics and microwave engineering with particular reference to the theory, design and computer modeling of microwave tubes. He is the author of a number of papers, two textbooks on electromagnetics, and a set of video lectures on microwave tubes.

Elaine M. Ball received a B.Eng. degree in electrical and electronic engineering in 1991 and a Ph.D. degree (on Magnetron Frequency Twinning) in 1996, both from Lancaster University, Lancaster. She worked at Lancaster University as a Research Assistant for three years before joining EEV as a TWT Development Engineer. She is currently working as a Product Reliability Engineer at Hewlett Packard, Bristol.

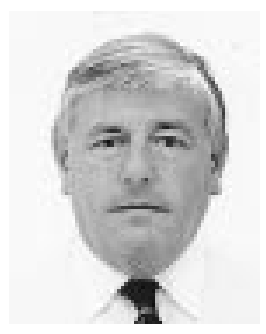

Michael B. Brady joined English Electric Valve Company in 1960 as a Student Apprentice and gained an H.N.D in electrical engineering from Mid Essex Technical College followed by I.E.E part 3 success in 1964. From 1964 to 1970, he worked on the design and manufacture of magnetrons for marine and military radars and for industrial heating. From 1970 to 1985 as a Principal Engineer responsible for a number of research and development projects including multipactor tuning of magnetrons, coaxial magnetrons and a new range of marine magnetrons. In 1985 he became a Technical Manager responsible for the development of phase locked magnetrons and millimeter magnetrons up to $95 \mathrm{Ghz}$ and in 1989, the Manager of a department manufacturing and developing magnetrons for marine airborne and surveilance radars. He is now a Chief Engineer and principal interest is very high power magnetrons at $9 \mathrm{GHz}$ and $3 \mathrm{GHz}$ for use with medical and industrial linear accelerators. 